EPJ Web of Conferences 61, 06003 (2013)

DOI: $10.1051 /$ epjconf/ 20136106003

(C) Owned by the authors, published by EDP Sciences, 2013

\title{
Analyzing polarization swings in 3C 279
}

S. Kiehlmann",a , T. Savolainen ${ }^{1}$, S. G. Jorstad ${ }^{2,3}$, K. V. Sokolovsky ${ }^{4,11}$, F. K. Schinzel ${ }^{5}$, I. Agudo A,2,7, A. A. Arkharov $^{8}$, E. Benítez ${ }^{9}$, A. Berdyugin ${ }^{10}$, D. A. Blinov ${ }^{3}$, N. G. Bochkarev ${ }^{11}$, G. A. Borman ${ }^{12}$, A. N. Burenkov ${ }^{13}$, C. Casadio $^{6}$, V. T. Doroshenko ${ }^{14,12}$, N. V. Efimova ${ }^{3}$, Y. Fukazawa ${ }^{15}$, J. L. Gómez ${ }^{6}$, V. A. Hagen-Thorn ${ }^{3,16}$, J. Heidtt ${ }^{17}$, D. Hiriart ${ }^{18}$, R. Itoh ${ }^{15}$, M. Joshi' ${ }^{2}$, G. N. Kimeridze ${ }^{19}$, T. S. Konstantinova ${ }^{3}$, E. N. Kopatskaya ${ }^{3}$, I. V. Korobtsev ${ }^{20}$, Y. Y. Kovalev ${ }^{4,1}$, T. Krajci ${ }^{21}$, O. Kurtanidze ${ }^{19,17}$, S. O. Kurtanidze ${ }^{19}$, V. M. Larionov ${ }^{3,8,16}$, E. G. Larionova ${ }^{3}$, L. V. Larionova ${ }^{3}$, E. Lindfors ${ }^{22}$, J. M. López ${ }^{18}$, A. P. Marscher ${ }^{2}$, I. M. McHardy ${ }^{23}$, S. N. Molina ${ }^{6}$, D. A. Morozova ${ }^{3}$, S.V. Nazarov ${ }^{12}$, M. G. Nikolashvili ${ }^{19}$, K. Nilsson ${ }^{22}$, N. G. Pulatova ${ }^{12}$, R. Reinthal ${ }^{10}$, A. Sadun ${ }^{24}$, S. G. Sergeev ${ }^{12}$, L. A. Sigua $^{19}$, M. Sorcia ${ }^{9}$, O. I. Spiridonova ${ }^{13}$, L. O. Takalo ${ }^{10}$, B. Taylor ${ }^{2,25}$, I. S. Troitsky ${ }^{3}$, L. S. Ugolkova ${ }^{11}$, J. A. Zensus ${ }^{1}$, and V. E. Zhdanova ${ }^{13}$

${ }^{1}$ Max-Planck-Institut für Radioastronomie, Auf dem Hügel, 69, D-53121, Bonn, Germany

${ }^{2}$ Institute for Astrophysical Research, Boston University, 725 Commonwealth Avenue, Boston, MA 02215, USA

${ }^{3}$ Astronomical Institute, St. Petersburg State University, Universitetskij Pr. 28, Petrodvorets, 198504 St. Petersburg, Russia

${ }^{4}$ Astro Space Center of Lebedev Physical Institute, Profsoyuznaya 84/32, 117997, Moscow, Russia

${ }^{5}$ Department of Physics and Astronomy, University of New Mexico, Albuquerque NM, 87131, USA

${ }^{6}$ Instituto de Astrofísica de Andalucía, CSIC, Apartado 3004, 18080, Granada, Spain

${ }^{7}$ Joint Institute for VLBI in Europe, Postbus 2, 7990 AA, Dwingeloo, the Netherlands

${ }^{8}$ Main (Pulkovo) Astronomical Observatory of RAS, Pulkovskoye shosse, 60, 196140, St. Petersburg, Russia

${ }^{9}$ Instituto de Astronomía, Universidad Nacional Autónoma de México, 04510 México DF, México

${ }^{10}$ Tuorla Observatory, Department of Physics and Astronomy, University of Turku, Väisäläntie 20, 21500, Piikkiö, Finland

${ }^{11}$ Sternberg Astronomical Institute, M. V.Lomonosov Moscow State University, Universiteskij prosp. 13, Moscow 119991, Russia

${ }^{12}$ Crimean Astrophysical Observatory, P/O Nauchny Crimea 98409, Ukraine

${ }^{13}$ Special Astrophysical Observatory of the Russian AS, Nizhnij Arkhyz, Karachaevo-Cherkesia 369167, Russia

${ }^{14}$ Southern station of the Moscow Lomonosov State University, Moscow, Russia, P/O Nauchny, 98409 Crimea, Ukraine

${ }^{15}$ Department of Physical Sciences, Hiroshima University, Higashi-Hiroshima, Hiroshima 739-8526, Japan

${ }^{16}$ Isaac Newton Institute of Chile, St. Petersburg Branch, St. Petersburg, Russia

${ }^{17} \mathrm{ZAH}$, Landessternwarte Heidelberg, Königstuhl 12, 69117 Heidelberg, Germany

${ }^{18}$ Instituto de Astronomía, Universidad Nacional Autónoma de México, 22860 Ensenada BC, México

${ }^{19}$ Abastumani Observatory, Mt. Kanobili, 0301 Abastumani, Georgia

${ }^{20}$ Inst. of Solar-Terrestrial Physics, Lermontov st. 126a, Irkutsk p/o box 291, 664033, Russia

${ }^{21}$ Center for Backyard Astrophysics - New Mexico, PO Box 1351 Cloudcroft, NM 88317, USA

${ }^{22}$ Finnish Centre for Astronomy with ESO (FINCA), University of Turku, Väisäläntie 20, FI-21500 Piikkiö, Finland

${ }^{23}$ Department of Physics and Astronomy, University of Southampton, Southampton, SO17 1BJ, United Kingdom

${ }^{24}$ Department of Physics, University of Colorado Denver, CO, USA

${ }^{25}$ Lowell Observatory, Flagstaff, AZ 86001, USA

\begin{abstract}
Quasar 3C 279 is known to exhibit episodes of optical polarization angle rotation. We present new, well-sampled optical polarization data for 3C 279 and introduce a method to distinguish between random and deterministic electric vector position angle (EVPA) variations. We observe EVPA rotations in both directions with different amplitudes and find that the EVPA variation shows characteristics of both random and deterministic cases. Our analysis indicates that the EVPA variation is likely dominated by a random process in the low brightness state of the jet and by a deterministic process in the flaring state.
\end{abstract}

\section{Introduction}

Rotations of the electric vector position angle (EVPA) of linearly polarized radiation have been observed in various blazars since the 1960s [e.g., 1]. Proposed explanations include stochastic variation [e.g., 2], multi-component models [cf. 3], and bent trajectories of a moving shock in a tan-

\footnotetext{
a e-mail: skiehlmann@mpifr-bonn.mpg.de
}

gled [cf. 4] or structured [cf. 5] magnetic field. In 3C 279 a $300^{\circ}$ counter-clockwise rotation of the optical EVPA was observed in 2006/2007 and explained by an emission feature on a spiral path in a helical magnetic field [6]. In 2009 a $210^{\circ}$ rotation in the opposite direction was observed and explained by a global bend of the jet [7].

A major issue in analyzing polarization data is the $n \pi$ ambiguity of the measured EVPA. Low sampling rates 
Table 1. Observatories participating in optical polarimetric monitoring.

\begin{tabular}{lrl}
\hline \hline Institution & Tel. diam. & Filters \\
\hline Calar Alto, Spain & $220 \mathrm{~cm}$ & R \\
CrAO, Ukraine & $70 \mathrm{~cm}$ & R \\
Kanata, Japan & $150 \mathrm{~cm}$ & V \\
KVA, La Palma, Spain & $60 \mathrm{~cm}$ & white light \\
Liverpool, La Palma, Spain & $200 \mathrm{~cm}$ & V+R \\
Perkins, USA & $183 \mathrm{~cm}$ & R \\
San Pedro Mártir, Mexico & $84 \mathrm{~cm}$ & R \\
St. Petersburg, Russia & $40 \mathrm{~cm}$ & white light \\
Steward Observatory, USA & 154 and $229 \mathrm{~cm}$ & $5000-7000 \AA$ \\
\hline
\end{tabular}

may obscure actual, large rotations and complicate the reconstruction and interpretation of the actual EVPA variation. We discuss here a method to distinguish random from deterministic EVPA variation and apply this method to our data set of optical polarization observations of 3C 279 from November 2010 to August 2012.

\section{Data set and data processing}

The photometric R-band data set consists of 24 light curves provided by different observers and institutions. We combine these individual light curves into one assembled light curve after removing clear outlier data points, averaging intra-night data, and cross-calibrating the individual light curves. Optical polarimetry data are provided by nine observatories listed in Table 1 . Since frequencydependence of the EVPA in optical is relatively weak, we combine all optical EVPA data sets to solve for the $n \pi$ ambiguity.

\subsection{EVPA ambiguity}

Comparison of EVPA measurements is ambiguous. The measured properties are the same for an EVPA $\chi$ and the EVPA $\chi^{\prime}=\chi \pm n \cdot \pi$ with $n \in \mathbb{N}$; thus, EVPA rotations larger than $\pi$ cannot be determined unambiguously. The common procedure to solve for this ambiguity is to assume a smooth variation of the EVPA and to shift data points to minimize the difference between adjacent data points (see e.g., [7]). We employ two methods to shift EVPA data points:

EVPA shifting method 1 determines the median of the $N$ previous data points as a reference for an EVPA data point and calculates the absolute deviation between them.

EVPA shifting method 2 calculates the absolute deviation between two adjacent data points and subtracts the root summed squared errors:

$$
\Delta \chi_{\mathrm{est}}=\left|\chi_{i}-\chi_{i-1}\right|-\sqrt{\sigma^{2}\left(\chi_{i}\right)+\sigma^{2}\left(\chi_{i-1}\right)}
$$

Both methods shift an EVPA data point by $\pm n \pi$ if the deviation is larger than $\pi / 2$ with $n \in \mathbb{N}$ such that the deviation is minimized. The first method with large $N$ is robust against measurement errors, but it is likely to obscure real

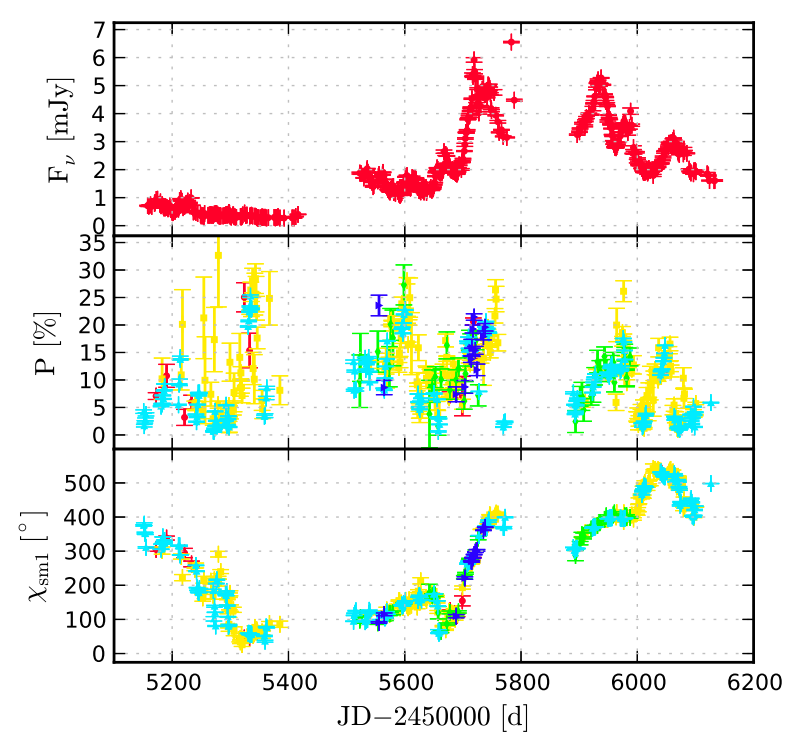

Figure 1. Optical polarization light curves of 3C 279. Upper panel: R-band flux density. Mid panel: Linear polarization fraction at V-band (red circles), R-band (yellow squares), V+R (green diamonds), 5000-7000 $\AA$ (pale blue up-sided triangles), white light (dark blue right-sided triangles). Lower panel: EVPA shifted with method 1 (symbols as in the second panel).

variation, if the data is not well sampled. With our worst data sampling of one data point each 20 days, $N=2$ must not be exceeded to reconstruct EVPA rotations rates up to $3.6^{\circ} / \mathrm{d}$ (measured with method 2 ). The fast rotations are sampled much better; the mean sampling rate allows for $N=16$. We choose $N=4$. The second method is expected to obscure real variation less frequently than method 1. With a sampling rate and errors similar to those of our observed data, both methods correctly reconstruct a simulated EVPA rotation of $500^{\circ} / 200 \mathrm{~d}$ with a probability of $98.5 \%$.

\subsection{Optical light curve and polarization}

Figure 1 shows the assembled R-band light curve, the degree of optical linear polarization, $P$, and the optical EVPA shifted with method $1, \chi_{\mathrm{sm} 1}$. The optical light curve shows little variation and low flux density, $F_{v}<1 \mathrm{mJy}$, before $\mathrm{JD}=2455400$. After the first seasonal gap the light curve covers a flaring state with an overall flux increase, two major flares, several smaller flares, and a flux decrease. During the first optical flare the flux density increases by a factor of 3 within 30 days. The mean linear polarization degree is $\langle P\rangle=12 \%$ with a standard deviation $\sigma(P)=8 \%$. Shifting the EVPA with method 1, we observe EVPA rotations in both directions with amplitudes up to $360^{\circ}$. A smooth $360^{\circ}$-rotation coincides with the first major optical flare. To test whether these EVPA rotations are of a random or a deterministic origin we use the scheme presented in the following section. 
Table 2. Simulation input parameters.

\begin{tabular}{llr}
\hline \hline time interval & $T$ & $200 \mathrm{~d}$ \\
mean time step & $\langle\Delta t\rangle$ & $3 \mathrm{~d}$ \\
min. time step: & $\Delta t_{\min }$ & $0.7 \mathrm{~d}$ \\
max. time step: & $\Delta t_{\max }$ & $21 \mathrm{~d}$ \\
$\Delta t$ power-law index: & $\alpha$ & -2 \\
error level: & $\sigma_{\text {errors }}$ & $4^{\circ}$ \\
\hline
\end{tabular}

\section{Discriminating random from deterministic EVPA variation}

Shifting the EVPA data points to solve the $n \pi$-ambiguity is based on the assumption that the EVPA actually rotates smoothly rather than jumps abruptly. This assumption leads to two questions. First, is it valid? If the underlying process is of a random origin, shifting EVPA data points artificially introduces rotations. And second, what does smooth mean for discretely sampled measurements? Jones et al. [2] have shown that random variations of the EVPA can produce rotations $>360^{\circ}$.

We define a quantitative measure of the smoothness of an EVPA curve and define a random process based on [2] and [8] to determine the probability of a random EVPA curve to show a large $\left(>180^{\circ}\right)$ and smooth rotation.

\subsection{A quantitative measure of smoothness}

The point-to-point variation of the EVPA curve is

$$
\left(\frac{\Delta \chi}{\Delta t}\right)_{i}=\frac{\chi_{i}-\chi_{i-1}}{t_{i}-t_{i-1}}
$$

in units of degrees per time unit. The mean of the pointto-point variation

$$
m=\left\langle(\Delta \chi / \Delta t)_{i}\right\rangle
$$

indicates a secular trend in the data. For a linear increase or decrease of the EVPA this value equals the slope of the linear regression. A point-to-point variation on the order of the trend indicates a smooth variation; whereas a strong deviation from the trend indicates a point-to-point variation larger than the general trend. The point-wise deviation of the variation from the trend is calculated as

$$
s_{i}=\left(\frac{\Delta \chi}{\Delta t}\right)_{i}-\left\langle\left(\frac{\Delta \chi}{\Delta t}\right)_{i}\right\rangle .
$$

The mean over this deviation is used as an estimator for the smoothness of the EVPA curve with respect to a potential linear trend:

$$
s=\left\langle s_{i}\right\rangle=\left\langle\left(\frac{\Delta \chi}{\Delta t}\right)_{i}-\left\langle\left(\frac{\Delta \chi}{\Delta t}\right)_{i}\right\rangle\right\rangle .
$$

The smoothness estimator $s$ is the mean absolute deviation over the mean (MAD) of the point-to-point variation as defined in eq. 2. An EVPA curve with $s_{1}$ is considered smoother than a second curve with $s_{2}>s_{1}$.

\subsection{Q-U-Random walk process}

To simulate a stochastic variation of the EVPA we perform a random walk in Stokes-Q-U-plane. The model contains $N$ cells of equal intensity $I$. The number $N$ determines the mean degree of linear polarization

$$
\langle P\rangle=\sqrt{\frac{\pi}{4 N}} \cdot P_{\max }
$$

with $P_{\max }=75 \%$ [8].

Each cell contains a uniform, but randomly oriented magnetic field. We draw random samples from uniform distributions of Stokes $Q_{i} \in[-1,+1]$ and $U_{i} \in[-1,+1]$ and normalize $Q_{i}$ and $U_{i}$ with the factor $P_{\max } / \sqrt{Q_{i}^{2}+U_{i}^{2}}$; with $i \in[1, N]$. The sums $Q=\sum_{i}^{N} Q_{i}$ and $U=\sum_{i}^{N} U_{i}$ determine the integrated degree of linear polarization, $P$, and electric vector position angle, $\chi$ :

$$
\begin{aligned}
& P=\frac{\sqrt{Q^{2}+U^{2}}}{I} \\
& \chi=\frac{1}{2} \arctan \frac{U}{Q}+n \frac{\pi}{2} \\
& \text { with } n= \begin{cases}1, & \text { if } Q<0 \\
0, & \text { otherwise }\end{cases}
\end{aligned}
$$

The variation of the polarization properties $P$ and $\chi$ is determined by the number of cells $N_{\text {var }}$ that change the magnetic field orientation in each mean time step $\langle\Delta t\rangle$ [8]. The fraction of cells $X_{\langle\Delta t\rangle}$ varying per mean time step $\langle\Delta t\rangle$ is estimated by the standard deviation $\sigma(P)$ of the degree of linear polarization:

$$
\frac{N_{\mathrm{var}}}{N}=X_{\langle\Delta t\rangle} \sim \frac{\sigma(P)}{\langle P\rangle} .
$$

For modeled time steps $\Delta t$ we scale the fraction of changing cells linearly with the time step:

$$
\begin{aligned}
X_{\Delta t} & =\frac{\Delta t}{\langle\Delta t\rangle} X_{\langle\Delta t\rangle} \\
N_{\mathrm{var}}^{\Delta t} & =X_{\Delta t} \cdot N \text { rounded to integer } \\
\text { with } N_{\mathrm{var}}^{\Delta t} & \leq N
\end{aligned}
$$

We define two slightly different random walk processes.

Simple random walk process: Each time step $N_{\text {var }}$ cells are selected randomly. The Stokes vectors of this subset of cells are reset randomly as described before.

Shock random walk process: To simulate a shock passing through a turbulent jet, the cells are numbered. Each time step the cells $i=1 . . N_{\text {var }}$ are deleted and $N_{\text {var }}$ new cells with randomized Stokes vectors are appended [e.g., 2, 8].

The observed $\langle P\rangle$ corresponds to $N=31$. The number of cells changed each mean time step is $N_{\mathrm{var}}=x \cdot N=$ 21. Averaged over all simulations the resulting standard deviation of the linear polarization degree is $\langle\sigma(P)\rangle=6 \%$, smaller than the measured $8 \%$. Even with the maximum 
Table 3. Simulation results for the simple and shock random walk process: frequencies of EVPA rotation amplitudes, smoothness estimators, EVPA trends, their mean over all simulations, and the frequency of both shifting methods leading to the same EVPA curve.

\begin{tabular}{|c|c|c|c|c|}
\hline \multirow[b]{2}{*}{ EVPA rotation amplitude $A_{\chi}$ : } & \multicolumn{2}{|c|}{ Simple random walk process } & \multicolumn{2}{|c|}{ Shock random walk process } \\
\hline & Method 1: & Method 2 & Method 1: & Method 2 \\
\hline$A_{\chi}>180^{\circ}:$ & $99.50 \%$ & $99.50 \%$ & $99.75 \%$ & $99.75 \%$ \\
\hline$A_{\chi}>360^{\circ}:$ & $42.49 \%$ & $42.49 \%$ & $50.5 \%$ & $50.5 \%$ \\
\hline$A_{\chi}>720^{\circ}:$ & $0.41 \%$ & $0.41 \%$ & $0.90 \%$ & $0.90 \%$ \\
\hline \multicolumn{5}{|l|}{ smoothness estimator $s$ : } \\
\hline$s<6^{\circ} / \mathrm{d}:$ & $>0 \%$ & $>0 \%$ & $>0 \%$ & $>0 \%$ \\
\hline$s<8^{\circ} / \mathrm{d}:$ & $0.008 \%$ & $>0 \%$ & $0.022 \%$ & $>0 \%$ \\
\hline$s<10^{\circ} / \mathrm{d}$ & $0.18 \%$ & $0.44 \%$ & $0.08 \%$ & $0.22 \%$ \\
\hline$s<20^{\circ} / \mathrm{d}:$ & $79 \%$ & $98 \%$ & $78 \%$ & $98 \%$ \\
\hline$\langle s\rangle=$ & $17.5^{\circ} / \mathrm{d}$ & $15.1 \% \mathrm{~d}$ & $17.7^{\circ} / \mathrm{d}$ & $15.1 \% \mathrm{~d}$ \\
\hline \multicolumn{5}{|l|}{ EVPA trend $m$ : } \\
\hline$|m|>1 \% / \mathrm{d}$ & $66 \%$ & $66 \%$ & $69 \%$ & $69 \%$ \\
\hline$\chi_{\mathrm{sm} 1}=\chi_{\mathrm{sm} 2}$ & \multicolumn{2}{|c|}{$1.28 \%$} & \multicolumn{2}{|c|}{$0.87 \%$} \\
\hline
\end{tabular}

Table 4. Smoothness estimator $s$ (errors in parantheses) and shifting consistency of the EVPA of 3C 279 in four observation epochs.

\begin{tabular}{llrr}
\hline \hline epoch & JD-2450000 & $s\left[^{\circ} / \mathrm{d}\right]$ & $\chi_{\mathrm{sm} 1}=\chi_{\text {sm2 }}$ \\
\hline I & $5150-5310$ & $32(5)$ & no \\
II & $5310-6050$ & $5.0(4)$ & yes \\
IIb & $5660-5760$ & $4.4(5)$ & yes \\
III & $6050-6110$ & $10.5(8)$ & yes \\
\hline
\end{tabular}

number of cells changing, $N_{\mathrm{var}}=N$, the averaged standard deviation of the linear polarization degree is $\langle\sigma(P)\rangle<8 \%$. This already indicates that the polarization curve of $3 \mathrm{C} 279$ is not produced by a stochastic process, at least not with one having the probability density functions that are used in our simulations.

The mean time step of our observations is $\langle\Delta t\rangle=3 \mathrm{~d}$. The simulation time series is constructed randomly with time steps $\Delta t$ following a power law distribution $\mathrm{P}(\Delta t) \propto$ $\Delta t^{\alpha}$ with $\alpha<-1$, within the limits $\left[\Delta t_{\min } ; \Delta t_{\max }\right]$. This simulates a time step distribution similar to the observed data. The EVPA is then calculated following either the simple random walk process or the shock random walk process. Simulated measurement errors are set randomly following a Gaussian distribution with standard deviation, $\sigma_{\text {errors }}$. The resulting EVPA is modified with both shifting methods. For each modified EVPA curve we determine the amplitude of variation, $A_{\chi}=\chi_{\max }-\chi_{\min }$, and calculate the smoothness estimator, $s$, and EVPA trend, $m$.

The simulation input parameters are shown in Table 2 . Large EVPA rotations $\left(>180^{\circ}\right)$ have been observed at the time scale of days to 100 days [e.g., 6, 7, 9]. We simulate time intervals of $T=200 \mathrm{~d}$, to measure the frequency of large rotations within this time scale. We run the simulation 1000000 times for both random walk processes.

\subsection{Simulation results}

Table 3 lists the frequencies of EVPA amplitudes $A_{\chi}$, smoothness estimators $s$, EVPA trends $m$ and the corresponding means over all simulations for both random walk processes and both EVPA shifting methods. The last row shows the probability that both EVPA shifting methods give the same result. The main simulation results are:

- Large EVPA rotations of $>360^{\circ}$ in less than $200 \mathrm{~d}$, as observed in our data, are common in random process based EVPA curves. The simple and the shock random walk processes produce rotations of that order with a probability of $43 \%$ and $51 \%$, respectively.

- Only in fewer than $1.3 \%$ of the simulations do both EVPA shifting methods result in EVPA curves that are consistent with each other.

- A smoothness estimator $s<8 \%$ d occurs less than 30 times in 1.000.000 simulations and $s$ is larger than $10 \%$ d with a probability $>99.7 \%$.

\section{EVPA variation in $3 C 279$}

Figure 2 shows a comparison of the measured polarization variation of 3C 279 (left plot) and one simulation over the same time interval as the observation, based on the simple random walk process (right plot). We make four general observations: 1) The observed polarization degree and EVPA $\left(180^{\circ}\right.$ interval) variation is less erratic than the simulated polarization degree and EVPA variation. 2) This simulation shows an EVPA variation amplitude of the same order as the observed, but 3) the simulated EVPA curve is less smooth than the observed one. 4) The smoothness of the observed EVPA curve is shown quantitatively in the small scatter of the point-to-point variation (eq. 2) compared to the simulation. For a more detailed and quantitative description we divide the observation time into four epochs. Details are listed in Table 4.

Epoch I covers the major clockwise rotation of the EVPA. The smoothness estimator is significantly higher than the mean smoothness estimator of the random walk process simulations. The two EVPA shifting methods give inconsistent results. Shifting method 1 gives a total rotation of $\sim 350^{\circ}$, method 2 of $\sim 550^{\circ}$. Both the high smoothness estimator and the shifting inconsistency point to a possible random process origin of the EVPA variation during epoch I. 

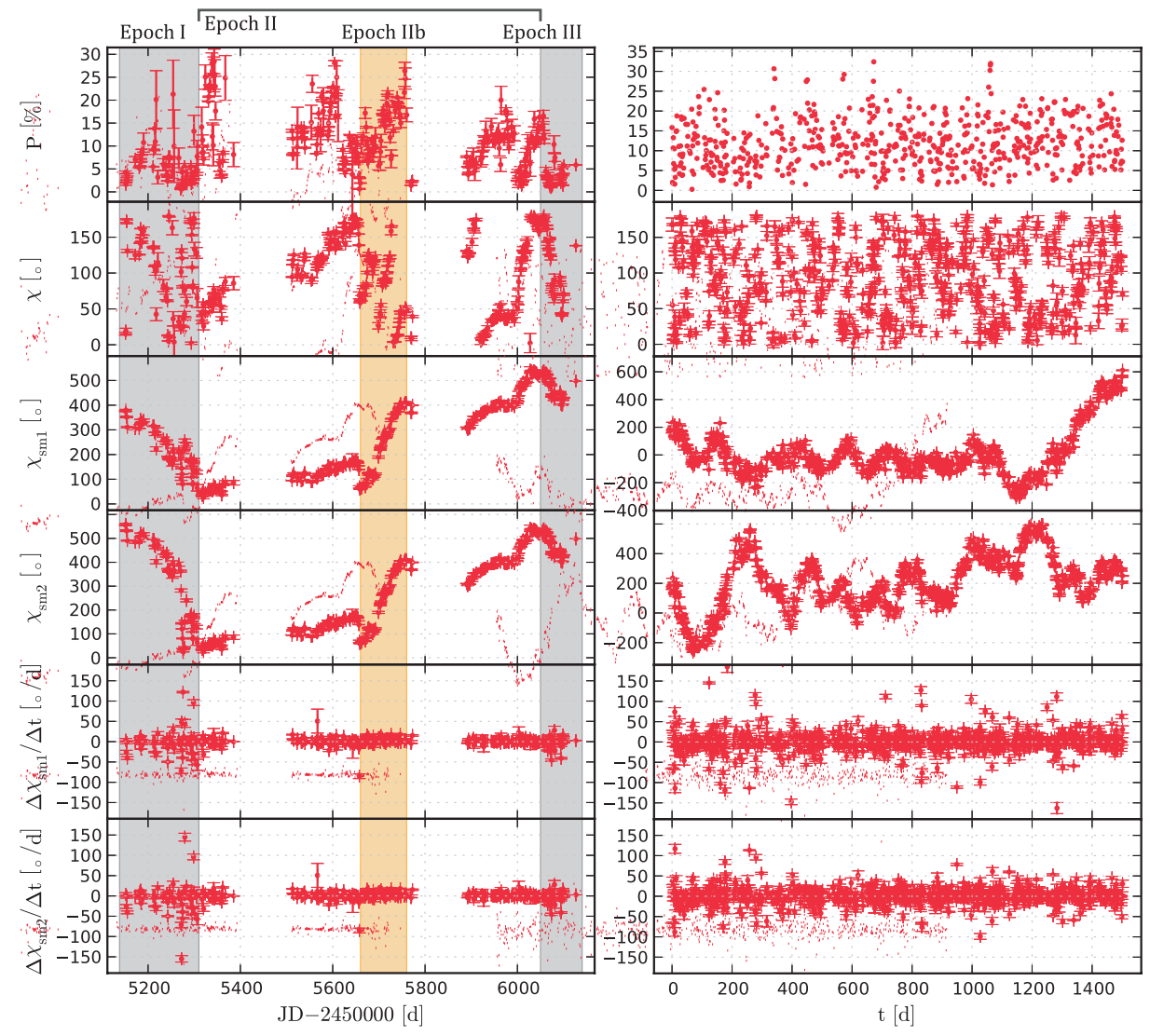

Figure 2. Polarization variation of $3 \mathrm{C} 279$ (left plot) and a simple random-walk process simulation (right plot). The panels show from top to bottom: degree of linear polarization, EVPA in the 180॰ interval, EVPA shifted with method 1, EVPA shifted with method 2, point-to-point variation (eq. 2) of the EVPA shifted with method 1 and method 2.

Epoch II covers a general EVPA counter-clockwise rotation with changing slope that includes one sharp EVPA decrease of $120^{\circ}$ in 4 days around JD2455655 and possibly a second decrease during the observation gap around JD2455800. The shifting results are consistent and the smoothness estimator is $s=5.0(4)$. Our simulation gives a probability of $<10^{-6}$ for creating EVPA variation with these characteristics from a random process. Epoch IIb is a subset of epoch II that shows a smooth $(s=4.4(5))$, counter-clockwise EVPA rotation of $360^{\circ}$ in $\sim 100$ days. During epoch III the EVPA decreases. The shifting methods are consistent, but the smoothness estimator increased with respect to the previous epoch.

This analysis indicates that two different processes affect the EVPA variation. During epoch I the jet is in a low state $\left(F_{v}<1 \mathrm{mJy}\right.$ (R-band)) and the EVPA variation likely originates from random fluctuations of the net B-field orientation in a turbulent emission region. At the end of epoch I - around JD2455350 - the degree of linear polarization increases by a factor of $2-5$. A new emission feature likely emerges in the jet. This new feature dominates the jet emission, increasing by a factor of $\sim 20$ from epoch 1 to the flare peak, and causes a deterministic EVPA rotation coinciding with flaring epoch 2. Different processes can explain a deterministic EVPA variation: 1) Abdo et. al. [7] explained the EVPA rotation of 3C 279 in February 2009 with a globally bending jet. Bends in different directions could explain the two-directional EVPA swings, but a rotation of at least $360^{\circ}$ requires the jet to follow a helical path. 2) Two orthogonally polarized emission features shift the integrated EVPA, when one of the features fades in or out. This process can rotate the EVPA in both directions, but only with amplitudes $<90^{\circ}$ [3]. 3) Two models are based on a jet emission feature - possibly a moving shock - that does not fill the full jet crosssection and moves along a helical or bent streamline. In one of them, the magnetic field can be tangled and the moving shock compresses and partially orders the tangled magnetic field [4]. The rotation of the EVPA is then coupled to the bent motion of the shock front $[10,11]$. In the other one the magnetic field is ordered - either helical or toroidal - and the feature 'highlights' different parts of the magnetic field, resulting in an EVPA swing [5, 12]. Both of the latter models are expected to produce uni-directional EVPA swings only, as the rotation is coupled to the motion of the feature within the jet cross-section. If the emission feature is ejected from the accretion disk the twist of the feature originates in the angular velocity of the disk and is not expected to change direction. However, our first modeling results - in preparation - indicate that two-directional EVPA rotations could indeed be reproduced in the framework of an emission feature following a helical trajectory in a helical magnetic field, assuming angular momentum conservation in a jet that is opening up downstream.

During epoch III the flux decreases, suggesting that the emission feature fades out and the random variations in the low state jet start to dominate again, consistent with the increase of the smoothness estimator.

\section{Conclusions}

When 'solving' the $n \pi$-ambiguity, one faces the danger of wrongly reconstructing the actual EVPA curve and misin- 
terpreting the data. Poor sampling rates may obscure large rotations. And shifting data points by multiple times of $\pi$ may artificially introduce large rotations that actually are random and non-directed variations.

We present a method to distinguish between possibly random EVPA variation and deterministic EVPA variation. We run Monte Carlo simulations of a random walk in Stokes-Q-U-space, adjusted to reproduce the mean and standard variation of the linear polarization degree that we observe, with a randomized sampling rate, that follows the same characteristics as our data. The introduced smoothness estimator quantifies the smoothness of the curve. The simulations of different kinds of random walk processes give us a threshold for the smoothness estimator. We can exclude these models at a certain confidence level, if the smoothness estimator of the observed data is smaller than the threshold. We point out that the threshold depends on the data sampling characteristics and the number of modeled cells, and thus the quoted values are only valid for our data set. The smoothness estimator threshold and the (in)consistency of the two presented shifting methods are two indicators to distinguish between possibly random and deterministic EVPA variation.

3C 279 shows both characteristics in different states. In the low state the EVPA rotation is consistent with a stochastic process, possibly due to turbulence in the jet. In the flaring state the EVPA behaviour is likely deterministic and possibly dominated by a single emission feature. We exclude the model of a bent jet and the simple twocomponent model. A helical trajectory of the emission feature seems to be able to explain the EVPA rotation and modeling the EVPA curve in this scenario is in progress. Also, modeling will be needed to discriminate between the two possibilities of a tangled and shock compressed magnetic field or a structured (toroidal or helical) field.

Optical flux density and EVPA during the $360^{\circ}$ rotation (epoch IIb) show a high resemblance to the EVPA variation of PKS 1510-089 in March 2009 and BL Lac in September 2005, all coinciding with a general optical flaring episode and ending with sharp sub-flare [9, 12]. Thus, we observe the flare-related EVPA rotations not only in different objects, but in different classes of objects (quasars and BL Lacs). The $360^{\circ}$ rotation in $3 \mathrm{C} 279$ takes $110 \mathrm{~d}$. Assuming a Lorentz factor $\Gamma=15$, this time corresponds to a traveled distance of $\Delta r \approx 5 \cdot 10^{5}$ Schwarzschild radii (assuming a BH mass of $6 \times 10^{8} M_{\odot}[7$, and references therein]). If this emission feature is located in the acceleration and collimation zone, the mean Lorentz factor and traveled distance are likely smaller.

With an increasing interest in well-sampled polarization data [e.g., 9, 12] and new optical polarimetry monitoring projects like RовороL (http://robopol.org/), our analysis method may prove to be useful in discriminating between stochastic and deterministic EVPA rotations. As we observe two different processes in the low state and the flaring state of $3 \mathrm{C} 279$, we point out that it is mandatory not only to observe blazars triggered during flaring states, but also with good sampling during low states to fully un- derstand the structure of the magnetic field and the processes responsible for EVPA variation.

\section{Acknowledgements}

S.K. was supported for this research through a stipend from the International Max Planck Research School (IMPRS) for Astronomy and Astrophysics at the Max Planck Institute for Radio Astronomy in cooperation with the Universities of Bonn and Cologne. The research at Boston University was partly funded by NASA Fermi GI grant NNX11AQ03G. K.V.S. and Y.Y.K. are partly supported by the Russian Foundation for Basic Research grant 13-02-12103. N.G.B. was supported by the RFBR grant 12-02-01237a. St. Petersburg University team was supported by the RFBR grants 12-02-00452 and 12-02-31193. E.B., M.S. and D.H. thank financial support from UNAM DGAPAPAPIIT through grant IN116211-3. The research at the IAACSIC is supported by the Spanish Ministry of Economy and Competitiveness and the Regional Government of Andalucía (Spain) through grants AYA2010-14844 and P09-FQM-4784, respectively. The Calar Alto Observatory is jointly operated by the Max-Planck-Institut für Astronomie and the Instituto de Astrofísica de Andalucía-CSIC. Data from the Steward Observatory spectropolarimetric monitoring project were used. This program is supported by Fermi Guest Investigator grants NNX08AW56G, NNX09AU10G, and NNX12AO93G. We acknowledge the photometric observations from the AAVSO International Database contributed by observers worldwide and used in this research. This paper has made use of up-to-date SMARTS optical/nearinfrared light curves that are available at http://www.astro.yale. edu/smarts/glast/ [13].

\section{References}

[1] T.D. Kinman, ApJL 148, L53 (1967)

[2] T.W. Jones, L. Rudnick, H.D. Aller, M.F. Aller, P.E. Hodge, R.L. Fiedler, ApJ 290, 627 (1985)

[3] P.A. Holmes, P.W.J.L. Brand, C.D. Impey, P.M. Williams, P. Smith, et al., MNRAS 211, 497 (1984)

[4] T.V. Cawthorne, W.K. Cobb, ApJ 350, 536 (1990)

[5] S. Kikuchi, Y. Mikami, M. Inoue, H. Tabara, T. Kato, A\&A 190, L8 (1988)

[6] V.M. Larionov, S.G. Jorstad, A.P. Marscher, C.M. Raiteri, M. Villata, et al., A\&A 492, 389 (2008)

[7] A.A. Abdo, M. Ackermann, M. Ajello, M. Axelsson, L. Baldini, et al., Nature 463, 919 (2010)

[8] F.D. D'Arcangelo, A.P. Marscher, S.G. Jorstad, P.S. Smith, V.M. Larionov, et al., ApJL 659, L107 (2007)

[9] A.P. Marscher, S.G. Jorstad, V.M. Larionov, M.F. Aller, H.D. Aller, et al., ApJL 710, L126 (2010)

[10] K. Nalewajko, International Journal of Modern Physics D 19, 701 (2010)

[11] V.M. Larionov, S.G. Jorstad, A.P. Marscher, D.A. Morozova, D.A. Blinov, et al., ApJ 768, 40 (2013)

[12] A.P. Marscher, S.G. Jorstad, F.D. D’ Arcangelo, P.S. Smith, G.G. Williams, et al., Nature 452, 966 (2008)

[13] E. Bonning, C.M. Urry, C. Bailyn, M. Buxton, R. Chatterjee, et al., ApJ 756, 13 (2012) 